\title{
Mobile Dating Application for Blinds Based on Vocal Attractiveness
}

\author{
Sudhathai Mankar and Yachai Limpiyakorn ${ }^{+}$ \\ Department of Computer Engineering, Chulalongkorn University, Bangkok 10330, Thailand
}

\begin{abstract}
Vocal attractiveness is an important social evaluation. Attractive voices are judged to be more persuasive and more socially desirable. This paper thus proposes an approach of mate selection for blinds based on vocal attractiveness. To support the accessibility, the mobile application is developed using the framework of Android Application Standard Developer Interface Guidelines. The K-means algorithm is also used to partition the data of voice pitch into three clusters of tones of voice. Once the blind users hit the intriguing voice, they can interact with the voice owner via the instant messaging service provided by mobile devices.
\end{abstract}

Keywords: vocal attractiveness, k-means, mobile application, blind date, accessibility.

\section{Introduction}

In general, a blind date means a social engagement between two people who have not previously met, usually arranged by a mutual acquaintance. However, nowadays, the great diversity of online dating services has emerged and displaced the traditional sources of dating partners, namely the workplace, families, and friends. These websites enable strangers to find and introduce themselves to new personal connections via personal computers or mobile devices.

Human voice functions as a means of communication in oral language. In non-linguistic role, it has the ability to convey biological information, like sex and age; social classifications such as race; and emotional states. Much of the vocal attractiveness literature emphasizes the role of mate selection and sexual preferences [1]. The study of Zuckerman et al. [2] reported that higher attractiveness, both facial and vocal, resulted in more positive impression. People independently perceive both men and women with more appealing voices as having more attractive faces [3] [4]. Oguchi and Kikuchi [5] examined the effects of voice and physical appearance on interpersonal attraction. The findings reported that the attractiveness of voice and physical appearance had independent effects on interpersonal attraction. Bright, generous voices, low vocal pitch and a small range of vocal pitch could increase interpersonal attraction.

Vocal attractiveness may serve as an important cue to mate selection. Hearing an appealing voice can heighten our feelings of attraction. In humans, voice pitch is thought to be a cue of underlying quality and an important criterion for mate choice [6] [7]. While men preferred higher pitched women's voices as marriage partners, women showed no overall preference for voice pitch in men [7]. This paper thus presents the vocal attractiveness based approach to developing the mobile dating application for blinds. Voice pitch is selected as an indicator of underlying mate quality. The application is implemented on Android platform using the framework of Android Application Standard Developer Interface Guidelines [8] to achieve minimum accessibility. Accessibility refers to the design of products, devices, services, or environments for people who experience disabilities.

\section{Research Methodology}

\footnotetext{
Corresponding author. Tel.: + 6682218 6959; fax: +66822186955

E-mail address:Yachai.L@chula.ac.th.
} 
Pitch refers to the frequency of the sound. It is the vibration rate that occurs usually measured in $\mathrm{Hz}$. (Hertz, or cycles per second). In this research, audio samples of 60 men and 60 women were recorded for 10 seconds each and stored into two datasets separated by gender, male or female. As shown in Fig. 1, each voice was then converted to its frequency. The verification step was carried out to filter out noisy data. The natural groupings of each cleaned dataset of voice frequency were then executed using K-means algorithm [9]. In literature, Ananthakrishna et al. [10] applied the simple k-means nearest neighbor classifier for the classification of pathological voices.

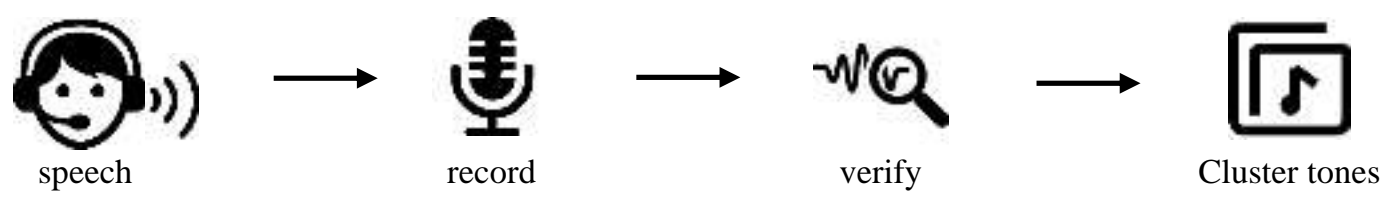

Fig. 1: Overview of research methodology.

The distributions of sample women and men's voice frequency data are shown in Fig. 2 and Fig. 3, respectively.

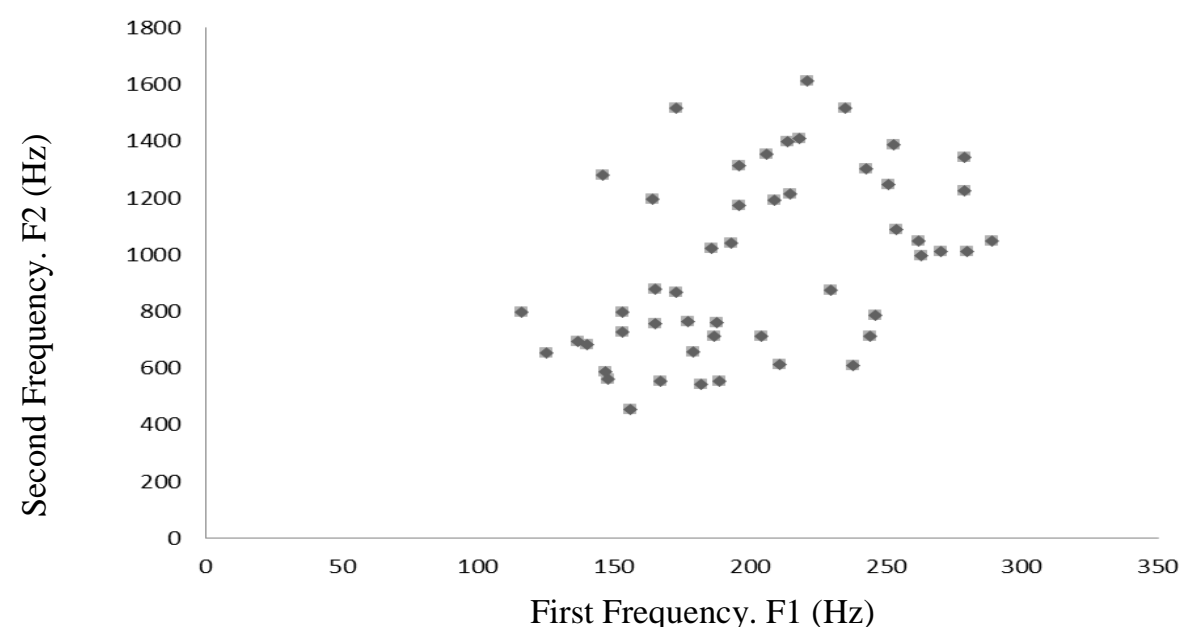

Fig. 2: Scatter diagram of 60 sample women's voice frequency data.

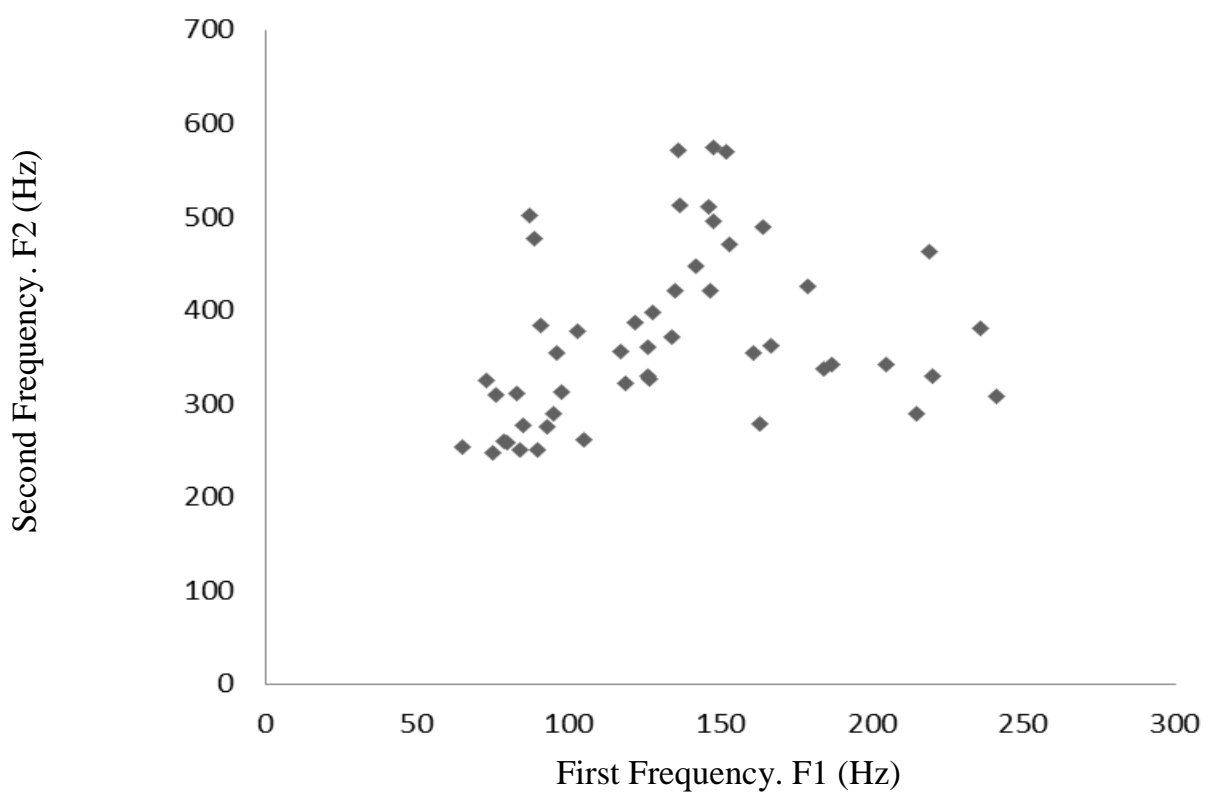

Fig. 3: Scatter diagram of 60 sample men's voice frequency data.

\section{K-means Clustering}


In this paper, $\mathrm{K}=3$ is defined for clustering the voice data of each gender into three tone categories: 1) low tone or bass, 2) mid tone, and 3) high tone, by chasing from left to right and from bottom to top. Fig. 4 and Fig. 5 illustrate the clusters resulted from executing the K-means algorithm on the two datasets shown in Fig. 2 and Fig. 3, respectively.

The member of each cluster will be randomly selected as the representative voice of each tone of voice when starting the application. In this work, the mobile application is developed conforming to the Android Application Standard Developer Interface Guidelines [8] to achieve minimum accessibility. For user interface controls, especially the image button and the checkbox, they will support the TalkBack (Android accessibility service). Fig. 6 illustrates an example of UI screen capture designed for blind assistive.

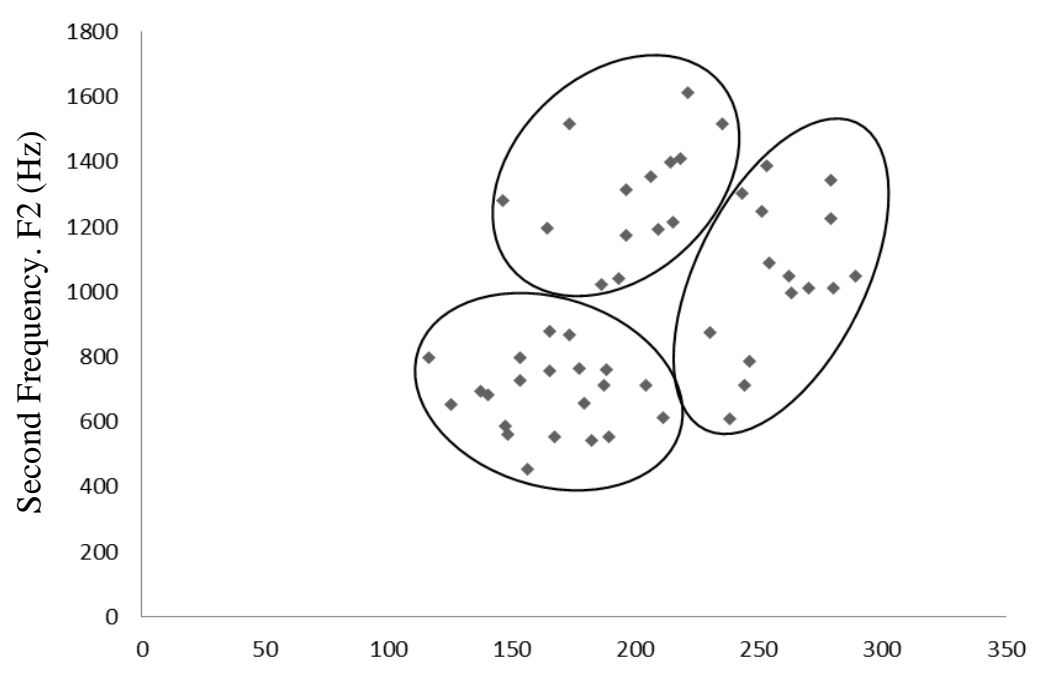

First Frequency. F1 (Hz)

Fig. 4: K-means segmentation of women voice tones.

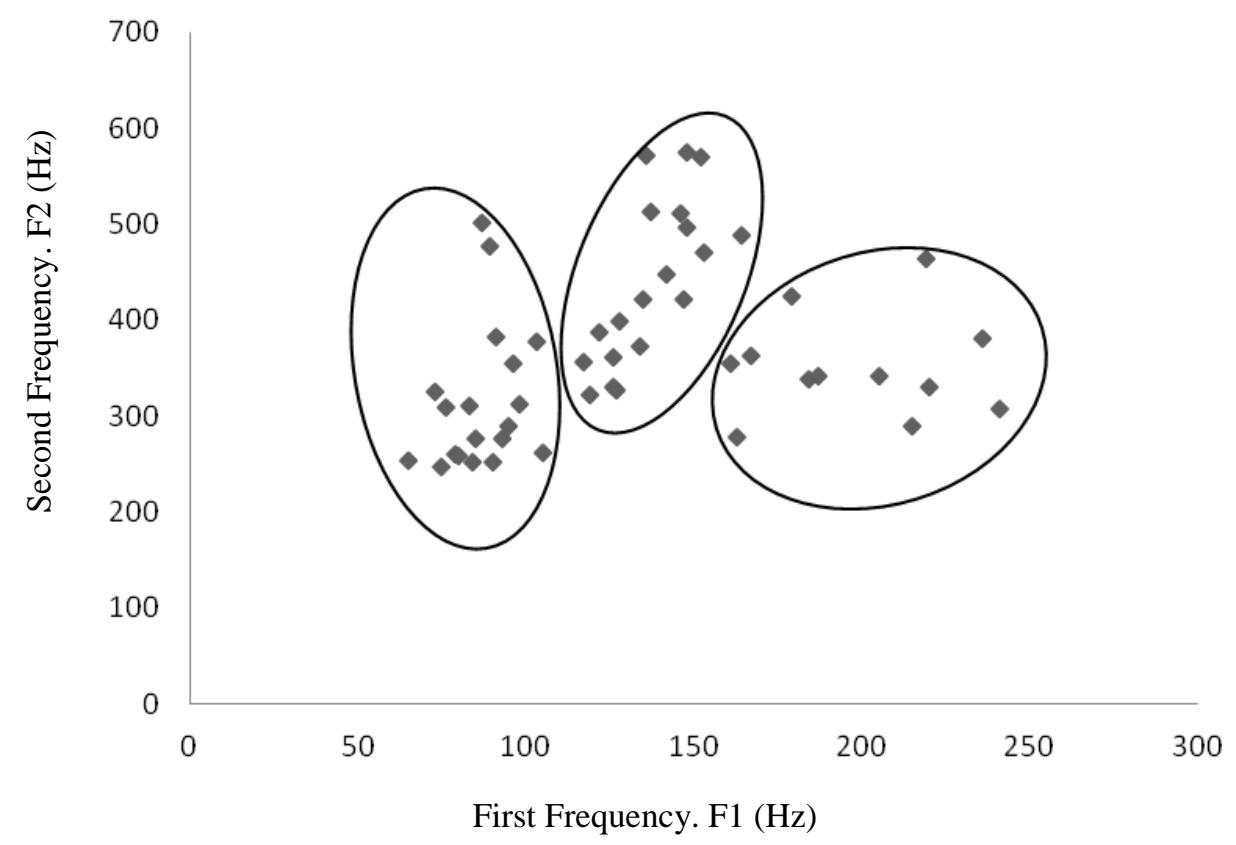

Fig. 5: K-means segmentation of men voice tones. 


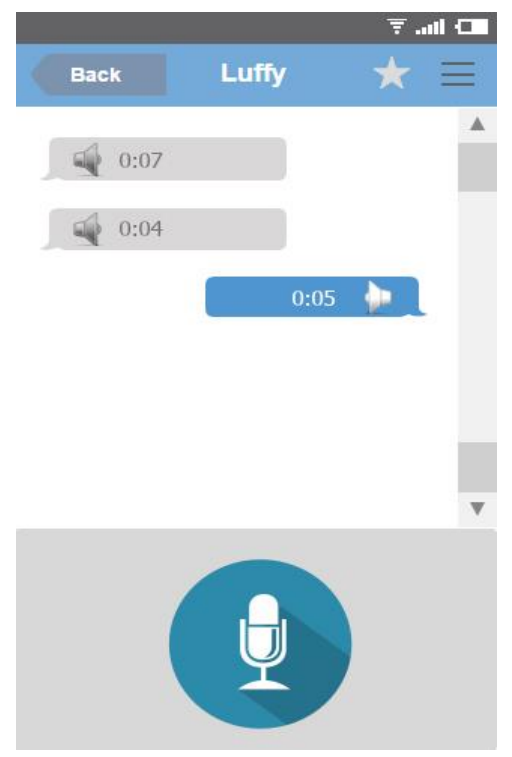

Fig. 6: Example of voice communication user interface.

\section{Conclusion}

People independently perceive that some voices are more attractive than others. Hearing an appealing voice can heighten their feelings of attraction. On the basis of listeners' judgments of attractive voices, this paper presents an approach to assist people, who are unable to see, for mate selection. Voice pitch is primarily selected as a dimension of underlying mate quality in humans. The K-means algorithm is applied for clustering the audio frequencies into groups of voice tones. The Android application has been developed providing the accessibility service TalkBack to help blind users interact with their devices. Further improvement on vocal segmentation would be carried out for better performance.

\section{References}

[1] M. Babel, G. McGuire, and J. King. Towards a more nuanced view of vocal attractiveness. PLoS One. 2014, 9(2): e88616.

[2] M. Zuckerman, K. Miyake and C.S. Elkin. Effects of Attractiveness and Maturity of Face and Voice on Interpersonal Impressions. Journal of Research in Personality. 1995, 29: 253-272.

[3] S.A. Collins, and C. Missing. Vocal and visual attractiveness are related in women. Anim. Behav. 2003, 65: 997

[4] T.K. Saxton, P.G. Caryl, and S.C. Roberts. Vocal and facial attractiveness judgments of children, adolescents and adults: the ontogeny of mate choice. Ethology. 2006, 112:1179-1185.

[5] T. Oguchi, and H. Kikuchi. Voice and interpersonal attraction. Japanese Psychological Research. 1997, 39(1): 56-61

[6] D.R. Feinberg. Are human faces and voices ornaments signaling common underlying cues to mate value? Evol. Anthropol.2008, 17: 112-118.

[7] C.L. Apicella, and D.R. Feinberg. Voice pitch alters mate-choice-relevant perception in hunter-gatherers. Proc Biol Sci. 2009, 276(1659): 1077-1082.

[8] “Accessibility | Android Developers" [Online]. Available: https://developer.android.com/guide/topics/ui/accessibility/index.html/ [Accessed: 21-Mar-2017].

[9] J. B. MacQueen. Some Methods for classification and Analysis of Multivariate Observations, Proc. of 5-th Berkeley Symposium on Mathematical Statistics and Probability. Berkeley, University of California Press. 1967, pp. 281-297.

[10] T. Ananthakrishna, K. Shama, and U. C. Niranjan. K-means nearest neighbor classifier for voice pathology. Proc. of the IEEE INDICON, 2004, pp. 352-354. 